\title{
LETTERS
}

\section{Updating our language around substance use disorders}

We thank Drs. Kumar and Rosenberg for enhancing care providers' knowledge of treatments for opioid abuse and its consequences. ${ }^{1}$ However, we need to be more precise in how we talk about the opioid crisis. Using the term "poisoning" instead of "overdose" would better reflect clinical reality and also change our subconscious thinking patterns to reduce the stigma faced by patients. We ought to be consistent and accurate in our portrayal of medical conditions, as it affects public attitudes and support for health policies. ${ }^{2}$ In the context of opioid abuse, we've heard many misconceptions about drug use and addictions, such as the idea that supervised injection sites promote substance abuse.

The term "overdose" connotes personal failure and responsibility, and is a remnant of the old psychosocial model of addiction. This term suggests that the patient a) knows the nature of the substance taken and $b$ ) has taken more than what she or he was tolerant to or intended to take. "Overdose" also implies that there is a correct dose, when none exists for use of illicit formulations.

In practice, opioid formulations sold in unregulated markets have variable and unknown potency, so dosing is impossible. In addition, people lose tolerance because of inconsistent use and concurrent illness, so dosing is variable at the individual level. For this reason, we dose opioids judiciously in acute care settings, with careful monitoring.

Consequently, we suggest that the more precise terms "poisoning" or "intoxication" should be used. These terms accurately label the acute medical illness and are physiologically and medicolegally aligned, corresponding with the International Statistical Classification of Diseases and Related Health Problems, 10th revision (ICD-10). Reorienting our language with respect to opioids is also compatible with public understanding regarding other substances: we say "alcohol poisoning" or "intoxication," and not "alcohol overdose."

With these more accurate terms, providers may be cued to consider and address the two separate health needs present: acute poisoning or intoxication, and the contributory conditions: uncontrolled pain, mental illness, drug dependence or addiction, etc.

As we update our understanding of substance use disorders from a psychosocial to a comprehensive medical model, it is imperative that we also update the language we use to accurately reflect the medical process in place. For the public, this can prevent moral judgments and confusion. Our hope is that using the right words can lead to more effective management of the opioid crisis as a public health emergency.

\section{Edward Xie MD}

Emergency Physician, University Health Network; Lecturer, Department of Family and Community Medicine, University of Toronto, Toronto, Ont.

\section{Samantha Green MD}

Family Physician, St. Michael's Hospital, University of Toronto, Toronto, Ont.

\section{Nitasha Puri MD}

Physician, Family Medicine and

Addictions, Vancouver Coastal Health;

Clinical Instructor, University of British

Columbia, Vancouver, BC

\section{Hasan Sheikh MD}

Emergency Physician, University Health Network; Lecturer, Department of Family and Community Medicine, University of Toronto, Toronto, Ont.

Cite as: CMAJ 2017 December 18;189: E1566. doi: 10.1503/cmaj.733490

\section{References}

1. Kumar T, Rosenberg H. Take-home naloxone. CMAJ 2017;189:E1192.

2. Kelly JF, Saitz R, Wakeman S. Language, substance use disorders, and policy: the need to reach consensus on an "addiction-ary." Alcohol Treat Q 2016; 34:116-23.

3. Behar E, Rowe C, Santos GM, et al. Primary care patient experience with naloxone prescription. Ann Fam Med 2016;14:431-6.

Competing interests: None declared. 